D. R. OWEN \& W. O. WiLLIAMS

\title{
Corrigendum: \\ On the Time Derivatives of Equilibrated Response Functions
}

Vol. 33, pp. 288-306 (1969)

The last equation in Section IV, described as "the defining equation of hypoelasticity" is incorrect. In the usual situation, where $\mathscr{X}$ is the collection of second-order tensors and $f(0)$ is non-singular, the formula should read

$$
\dot{\pi}(f)=A(\pi(f))\left[\dot{f}(0) f(0)^{-1}\right] .
$$

The third in the list of sufficient conditions that $\pi$ be hypoelastic must then be changed. Thus: $\pi$ is a hypoelastic function if

1) $\pi$ is rate-independent and smooth,

2) $a_{f}$ exists for every $f \in \mathscr{F}$,

3) there exists $A: \mathscr{Y} \rightarrow \mathscr{L}(\mathscr{X}, \mathscr{Y})$ such that

$$
a_{f}=A(\pi(f)) \circ R_{f(0)^{-1}} .
$$

Here for any $a \in \mathscr{X}, R_{a}$ is the right-multiplication operator:

$$
R_{a} b=b a, \quad b \in \mathscr{X} .
$$

Accordingly, the last italicized statement in the introduction should read: if the stress is given by a smooth rate-independent function, then the material is hypoelastic if and only if its instantaneous modulus is the composition of a function of the stress with right-multiplication by the inverse of the deformation gradient.

$$
\begin{gathered}
\text { Carnegie-Mellon University } \\
\text { Pittsburgh }
\end{gathered}
$$

(Received August 18, 1969) 